\title{
FRUSTRACIONES Y PARADOJAS DE PROYECTOS ASPIRACIONALES EN UN FRACCIONAMIENTO DE LA METRÓPOLIS DE GUADALAJARA
}

\author{
FRUSTRATION AND PARADOXES OF ASPIRATIONAL PROJECTS IN A
}

GATED COMMUNITY OF GUADALAJARA'S METROPOLITAN ARE

\section{David Foust Rodríguez*}

\section{RESUMEN}

El objetivo de este artículo fue mostrar las tensiones y paradojas de los proyectos aspiracionales de clase media en un fraccionamiento de la periferia metropolitana de Guadalajara. Siguiendo un diseño mixto, entre 2012 y 20I4, se realizó trabajo de campo en un fraccionamiento de Tlajomulco de Zúńiga, Jalisco. El posicionamiento de las personas que participaron en la investigación fue analizado mediante la triangulación de análisis de conglomerados y su correlato discursivo. El resultado de este análisis da cuenta de la heterogeneidad social y económica en estos fraccionamientos, de las aspiraciones, contradicciones, paradojas y frustraciones de hogares de clase media que buscan resistir el descenso social en una sociedad neocolonial con desigualdad persistente.

Palabras clave: Segregación residencial, fraccionamientos cerrados, clase media, proyectos aspiracionales, metrópolis contemporáneas latinoamericanas.

\section{ABSTRACT}

The goal of this article is to show the tensions and paradoxes of middle class aspirational projects in a gated community of Guadalajara's metropolitan area. Following a mixed method approach, between 2012 and 20I4, field research was undertaken in a gated community in Tlajomulco de Zúñiga, Jalisco. Participants' positioning analysis was done by cross-checking cluster analysis and the associated discourses. The result of this analysis is an account of the social and economic heterogeneity; and of middle class households' aspirations, contradictions, paradoxes and frustrations while trying to escape downward social mobility in a neocolonial society with persistent inequality.

Keywords: Residential segregation, gated communities, middle class, contemporary aspirational projects, Latin American metropolitan areas.

Recibido 7 de junio de 2019 y aceptado 30 de octubre de 2019

*Profesor-Investigador en el Instituto Tecnológico y de Estudios Superiores de Occidente (ITESO), sNi (nivel candidato). Doctor en Ciencias Sociales (Sociología) por la Universidad de Guadalajara. Miembro de la Red Mexicana de Investigación en Política Social (REMIPSO) y de la Red Nacional de Investigadores en Estudios Socioculturales de las Emocionales (RENISCE). Sus líneas de investigación son capital social, política social, emociones y cambio social en México y en Estados Unidos, emociones y apropiación social del espacio<foust@iteso.mx>. 


\section{INTRODUCCIÓN}

Las metrópolis ${ }^{1}$ contemporáneas están siendo la plasmación territorial de procesos de acumulación de capital, de «creación destructiva» (à la Schumpeter) y de reproducción de la desigualdad social (Harvey, 20I2). Contra las tendencias a la democratización del territorio, las élites configuran «ciudades cerradas», «microestados» vigilados y semiautárquicos, discontinuidades que rompen la utopía de las ciudades para todos (Graham y Marvin, 200I; Harvey, 20I2; Siqueiros, 2009a y b; Svampa, 2004). Configuran un modo urbano de ser y estar que da cuerpo a la segregación social (Calonge, 2013). Se trata de uno de los mecanismos más visibles para dar salida al problema de la absorción del exceso de capital (Harvey, 20I2, pp. 6-7), pero también de un proyecto de civilización, si es que podemos usar ese nombre. La clase alta se constituye en modelo a seguir e imitar para el conjunto de la sociedad, incluso se impone como tal; su modo de ser se ostenta como prescripción legítima de ser (Camus, 20I5, pp. 266-268; Camus, 20I8; Veblen, 2009/I899, p. 59).

Pese a su discursivo individualista, o muy a su propósito, las élites constituyen a los fraccionamientos cerrados como un dispositivo de "colectivismo práctico" para organizar la reproducción de su hábitat y de su habitus (Svampa, 2004, pp. 66-67, cit. a M. y M. Pinçon, 2000, pp. IO2-IO4; véase, también, Calonge, 2013; Bourdieu, 1996/1979). El modelo del fraccionamiento cerrado que pretende garantizar seguridad, comodidad, homogeneidad socioeconómica y cierta autosuficiencia en la provisión de los servicios permea todas las capas de la sociedad (Siqueiros, 2009a, p. 29). Pero, al parecer, es como un barco que hace agua en todos los estratos socioeconómicos en donde se está probando.

Particularmente problemática resulta esta pretensión civilizatoria en ciudades neocoloniales o neoestamentales, como la metrópolis de Guadalajara (Camus, 20I4, 2015 y 20I8). Esta región no es solo «difusa, fragmentada y claramente disfuncional» (Lara y Mateos, 2015, p. 165) en términos de

${ }^{\mathrm{r}}$ Una metrópolis es una «extensión territorial que incluye a la unidad política administrativa que contiene a la ciudad central, y las unidades contiguas a esta que tienen características urbanas, tales como sitios de trabajo o lugares de residencia de trabajadores dedicados a actividades no agrícolas y que mantienen una interrelación socioeconómica directa, constante e intensa con la ciudad central y viceversa» (Unikel, I976, p. II8). 
infraestructura física, sino también en términos sociales, económicos y políticos. El mecanismo causal más inmediato y reciente de esta disfunción puede ubicarse en la ausencia de una política de desarrollo urbano articulada y sensata (ibid.; Coulomb, 2007; Siqueiros, 2009a y b). Pero dar cuenta cabal de este proceso implica rastrear la trayectoria varias veces secular de la metrópolis de Guadalajara.

La hipótesis de Camus (2015) es que el rostro actual de esta «neoestamentización» en la modalidad de $\operatorname{cotos}^{2}$ exclusivos -varios de ellos ubicados en la periferia- es la continuación de un patrón histórico marcado por una colonialidad que nunca se ha ido, pero que ahora adquiere nuevos matices (ibíd., pp. 22-23). Esta neoestamentalización consiste en el establecimiento de facto de privilegios fundados en sí mismos y por la fuerza (Weber, 1967, cit. por Camus, 20I5, p. 2I) y en la simulación de pertenencia a la aristocracia local (Camus, 2018). Cabrales y Canosa (200I) reseñan la búsqueda de exclusividad en Guadalajara en varios episodios, uno de ellos, la garden city tapatía: Chapalita; y el más reciente capítulo, el establecimiento de fraccionamientos cerrados y exclusivos, los country clubs; los primeros aparecen desde los ańos sesenta.

Calonge (2013) y Camus (2015) han estudiado la dialéctica entre la configuración de la identidad y del espacio en un fraccionamiento residencial de clase alta en Zapopan. Camus (20I4) también ha estudiado los fraccionamientos de clase media que llama wannabe, tratando de sintetizar con esta palabra la pretensión de ascenso social de sus ocupantes. Retomando su propio trabajo, Svampa (2004, p. 67) destaca las dificultades a las que se enfrentan las clases medias cuando pretenden participar del proyecto de reproducción social de la clase alta: lo que se logra, acaso, es tratar de ampliar el círculo social para vincularse con sectores de clase media alta y alta (2004, p. 67). Pero el intento de «integración social hacia arriba» suele frustrarse por la falta de clausura social en el sistema. Se asigna un rol de filtro o regulador social a la infraestructura defensiva (véanse Hill, 2005; Jorquera, 20II; Calonge, 2013). Pero aquellos a los que se pensaba filtrar ya están adentro y no siempre se apegan a lo que se espera de ellos (Svampa, 2004).

${ }^{2}$ Camus (2015, p. I3) destaca que coto significa «defendido» y uso para referirse a este tipo de fraccionamientos residenciales cerrados es propio de Guadalajara. 
Coincidimos con Frisch, González y Steindhardt (20I7) cuando señalan que hace falta subrayar la falta de homogeneidad socioeconómica y política en estas urbanizaciones cerradas, especialmente en las de clase media; y estudiar las contradicciones sociopolíticas que implica este modelo en los estratos medios (en esta misma línea, véanse Svampa, 2004; Camus, 20I5). Este trabajo pretende contribuir a la discusión y comprensión de esta problemática mostrando la heterogeneidad social y económica al interior del fraccionamiento de clase media estudiado. También analizaremos las tensiones y contradicciones que se generan a causa de esta heterogeneidad y de la disputa por el estatus y la concreción de los proyectos aspiracionales.

\section{FRACCIONAMIENTOS WANNABE: VOCES DE SIRENAS Y BALSAS HUNDIDAS}

\section{ANTECEDENTES DEL ESTUDIO}

Lara y Mateos (2015, pp. I65-167) hacen notar que en las últimas tres décadas (entre 1980 y 20I0) la zona metropolitana duplicó su población, pero que prácticamente todo el crecimiento se localizó fuera de Guadalajara. De los municipios periféricos, el que más ha crecido ha sido Tlajomulco de Zúñiga, también sede de la «eclosión del urbanismo defensivo" (Cabrales, 2006, p. I4, cit. en Lara y Mateos, 20I5, p. I68) y del viviendismo (ibíd.), especialmente de los años noventa a la fecha. Este modelo de vivienda de interés social a bajo costo, pero también de baja calidad, frecuentemente ubicada en las periferias de las metrópolis y con fuertes implicaciones sociales por su falta de infraestructura, equipamiento urbano, parques, escuelas, vialidades, servicios de agua, drenaje, transporte, etcétera. Valle del Ascenso ${ }^{3}$ tiene la peculiaridad de ser un claro protagonista de la arquitectura defensiva propia de los fraccionamientos cerrados de clase media, pero está ubicado en una zona en la cual predominan los fraccionamientos semicerrados viviendistas, accesibles a los hogares de menores ingresos.

\footnotetext{
${ }^{3}$ Sirva este nombre ficticio para connotar algunos de los principales hallazgos de la investigación.
} 
Valle del Ascenso fue diseñado y construido por las mismas empresas que Valle Real, el muy exclusivo fraccionamiento estudiado por Calonge (2013) y Camus (2015); de hecho, tiene un diseño similar, pero ajustado a la capacidad de compra de hogares de clase media baja. Por su ubicación en el municipio de mayor crecimiento de la metrópolis, a un costado de una de las avenidas más saturadas en las horas pico del tránsito vehicular, y por estar rodeado de colonias y fraccionamientos viviendistas, Valle del Ascenso es un experimento natural para estudiar la dinámica de hogares que, con más recursos, hubieran intentado vivir en el cuadrante norponiente de la metrópolis, pero que tuvieron que conformarse con vivir en un fraccionamiento muy bonito, pero muy mal ubicado. Parece lógico pensar que esta situación se vuelva caldo de cultivo para procesos de atopia (sentirse ajeno en el propio espacio) y topofobia (Tuan, I974 y 1977; Lindón, 2005; Hiernaux y Lindón, 2004b).

También resultaba más accesible, pues durante el periodo 2009-2010 fui miembro de la mesa directiva del coto en donde vivo, en este fraccionamiento. Esta experiencia me permitió convivir con otros representantes y con mis vecinos, y tener un acceso privilegiado a sus opiniones y puntos de vista. Como es obvio, estar implicado también tenía el inconveniente epistemológico de la falta de distancia. Para hacer contrapeso a este inconveniente, implementé varios mecanismos de vigilancia: llevar un registro en una bitácora de investigación que luego era la base de un diálogo crítico constante con otros colegas; buscar intencionalmente datos que desafiaran mi punto de vista sobre los aspectos estudiados; ampliar el número de hogares de la encuesta para lograr una muestra más representativa.

Rodeado por fraccionamientos semicerrados de clase baja ubicados más allá del periférico, ${ }^{4}$ Valle del Ascenso (véase Imagen I y 2) nos recuerda aquello de «más vale ser cabeza de ratón que cola de león». Tiene barda perimetral, tres accesos con caseta de vigilancia privada y plumas para regular el acceso de los vehículos, lo cual no ha impedido los robos a casa-habitación, entre otros delitos. Cuenta con amplias y bien cuidadas áreas verdes, pozo

${ }^{4}$ Hiernaux y Lindón (2004a, p. 420 y sig., retomando a Capel, 200I) hacen notar que la periferia es heterogénea y no puede caracterizarse como terreno uniforme para ciudades-dormitorio. 
propio (aunque con intermitentes problemas para dar el servicio) y sistema de recolección de basura, administrados por una Asociación de Colonos mediante un sistema de cuotas bimestrales de 250 pesos (que, como en muchos otros cotos de este tipo, no pagan muchos de los hogares). La cuota no ha subido en diez años; es decir, su valor real ha caído aproximadamente 50\% (según la calculadora de inflación de INEGI). Puede ser un indicador de la insuficiente legitimidad de la Asociación de Colonos y del sistema de mercantilización de los servicios (jardinería, atención del pozo, seguridad), notas principales del régimen condominal en este tipo de fraccionamientos (véanse Jorquera, 20II; Simmel, I986, cit. en Svampa, 2004).

Imagen I. Fotografías tomadas en Valle del Ascenso entre junio y agosto de 2013.

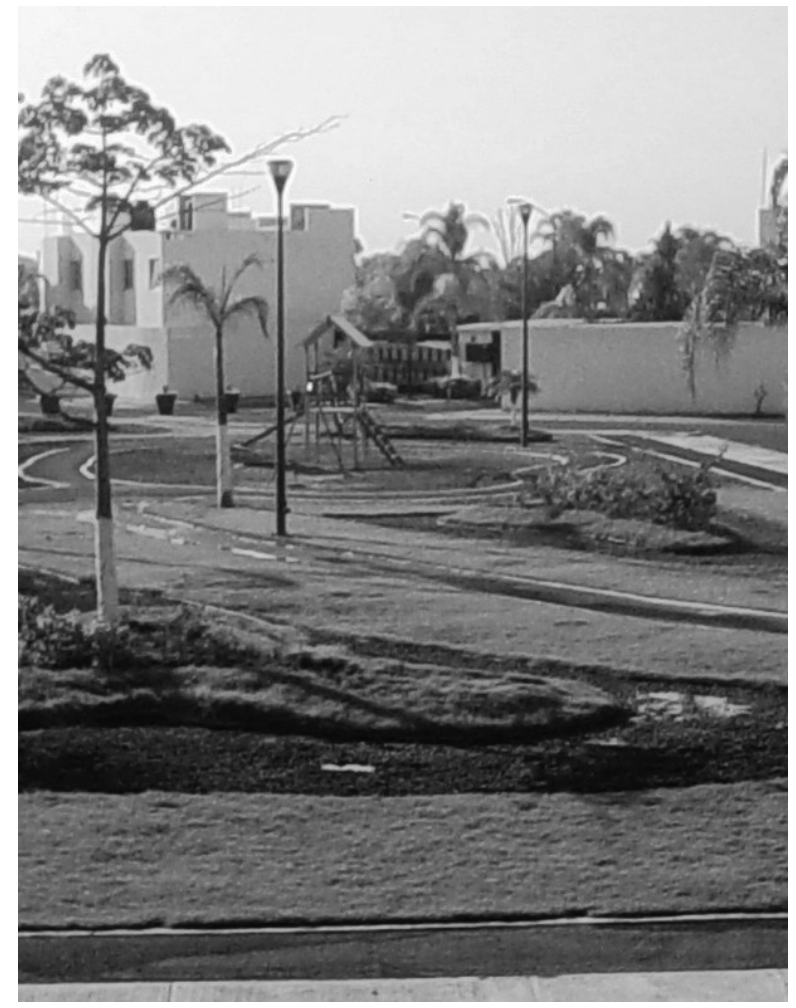


Imagen 2. Fotografias tomadas en Valle del Ascenso entre junio y agosto de 2013.

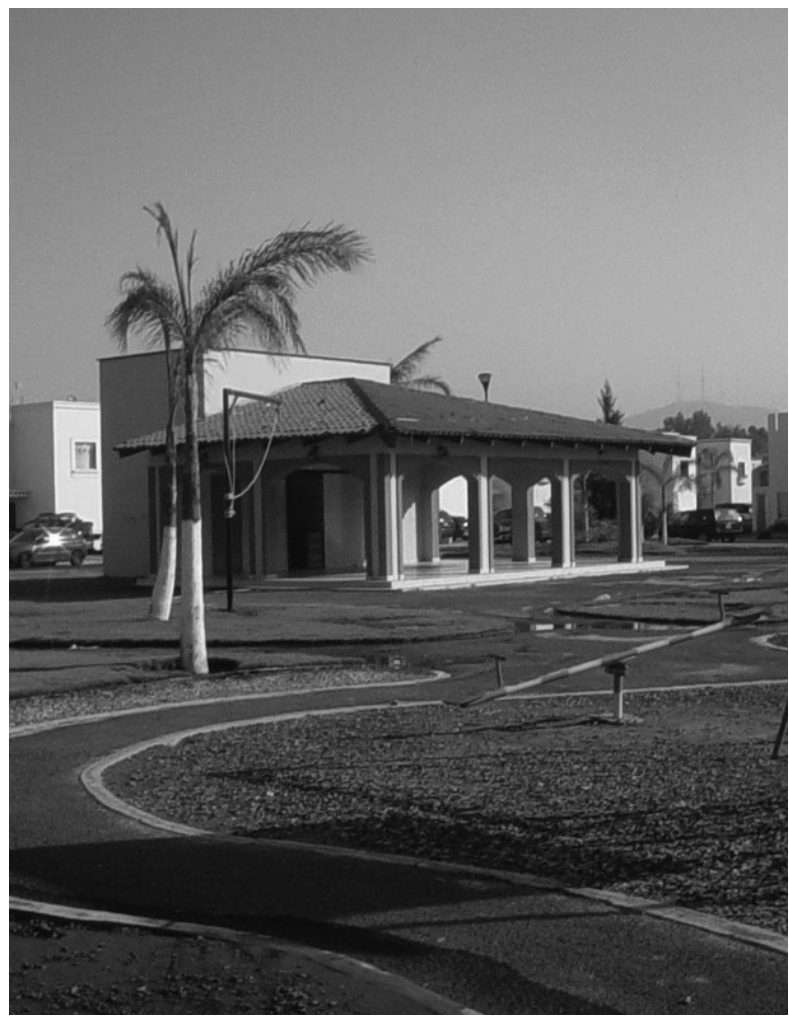

Fuente: Autoría propia.

En este fraccionamiento, 63\% de los encuestados son dueños de su casa (o están pagando su crédito hipotecario). Las viviendas tienen entre 85 y 125 metros cuadrados de construcción y tienen un precio de alrededor de 750 mil pesos. Poco menos de dos tercios de los hogares (6I.2\%, más del triple de la media nacional) fueron clasificados como no pobres y no vulnerables. 5 Los

5 Para seguir, en lo posible, los criterios oficiales para la medición multidimensional de la pobreza en México, publicados por el Consejo Nacional de Evaluación de la Política de Desarrollo Social (CONEVAL), se incluyeron preguntas sobre el acceso a servicios de salud, de sistemas de seguridad social, etcétera. Los ingresos se estimaron usando como proxy la escolaridad del perceptor principal del hogar, de manera similar a como se estima el nivel 
perceptores de estos hogares tienen niveles de escolaridad que oscilan entre la secundaria y la licenciatura. Comprar casa aquí es publicitado como adquirir un boleto para participar de la clase media: «¡Vida plena!», decía uno de los anuncios en las oficinas de ventas a la entrada del fraccionamiento; "Llegó el momento de crecer. Todo en tu colonia», dice otro letrero. Se anuncia que el lugar tiene sus propias escuelas y hasta su propio templo, y se destaca que tiene muchas y bonitas áreas verdes.

El canto de las sirenas está compuesto por estas notas: seguridad y comodidad accesible, ambiente familiar, "gente como uno", trampolín social (Camus, 20I4). Se trata del «imaginario de llegada: ascenso, exclusividad, sueños que se cumplen» (Arizaga, 2003, cit. en Frisch, González y Steinhardt, 20I7, p. 5).

Pero pronto desentonan las sirenas: no todos los colonos pagan sus cuotas; los espacios comunes son pocos o disputados; la música está a demasiado volumen; otros se estacionan en el parque o no levantan las heces de sus perros. El lugar está muy bonito, pero hay que salir para todo y los camiones siempre van llenos, además de que hay que atravesar la nueva frontera social: el Periférico. Termina uno llegando a la hora de dormir y saliendo antes de que salga el sol; y no siempre hay agua (algo muy similar a lo que describe Camus, 20I4).

Si los cotos de clase alta encubren una gran heterogeneidad bajo un discurso ideológico unificador (Camus, 2015, p. 26I), los fraccionamientos cerrados de clase media aun más (Camus, 20I4; Frisch, González, Steinhardt, 20I7). Como veremos, Valle del Ascenso es un caso que podríamos tomar como representativo, aunque con las particularidades que hemos mencionado, es decir: ser un fraccionamiento muy bonito, pero ubicado en la periferia de difícil tránsito de una metrópolis neoestamental.

En un texto clave para la sociología de las emociones, Kemper (1978) elabora una matriz sobre dos ejes, el estatus y el poder. De acuerdo con Kemper, y en consonancia con Weber (1946, p. 180, cit. por Kemper, 1978, pp. 32-33), «el estatus son los beneficios que los demás nos ofrecen libremente

socioeconómico cuando se usa la metodología de la Asociación Mexicana de Agencias de Investigación de Mercado (AMAI). 
(reconocimiento, afecto, honor, etc.); el poder es lo que extraemos de ellos por la fuerza. [...] A falta de estatus, se recurre al poder».

Young y Willmott (1962) analizaron un asentamiento, entonces nuevo, en el Este de Londres. Las casas son bonitas, pero todo queda lejos. En la tienda uno no se encuentra al vecino de toda la vida. Estos han quedado atrás, en los barrios pobres. Young y Willmott (1962, p. 318) subrayan que esta competencia se da, principalmente, con los anteriores vecinos: consiste en demostrar que ellos han logrado superarse, como suele decirse. Con los nuevos vecinos se establece una carrera para nivelarse hacia arriba con su nuevo estatus. Las personas toman como modelo a los vecinos que tienen más tiempo en la nueva colonia; si compran unas cortinas, ellos también; si ponen x o y plantas, ellos también (ibíd., pp. 32I-322). Para estar al día, se monitorean mutuamente. "No puedes tener algo nuevo porque ya lo quieren, te miran con celos» (ibíd., pp. 322). "Ansiedad por el estatus», le llama Botton (2004, p. 8, cit. en Camus, 20I4, p. I7). «Se refiere a un drama interior, una preocupación de 'no responder a los ideales de éxito establecidos por nuestra sociedad y que quizá por ello nos vemos despojados de dignidad y de respeto'.

Los otros no parecen «amigables», decían los participantes en la investigación de Young y Willmott (1962), y terminaban estableciéndose relaciones de competencia por estatus ante la falta de afecto mutuo. Pero he aquí una paradoja que nos recuerda la dialéctica del amo y el esclavo de Hegel (2006/1807): el estatus, para que sea genuino y surta sus efectos, debe ser otorgado libremente; ni puede ser arrancado por la fuerza, ni lo pueden dar aquellos que no son, también, reconocidos. El amo, al admitir que necesita del reconocimiento libre del esclavo, lo libera simbólicamente.

Pero la infraestructura física de los fraccionamientos cerrados de clase media no alcanza a ser suficiente para lograr una arquitectura defensiva eficaz o un filtro social que facilite la homogeneidad; es porosa (Frisch, González, Steinhardt, 20I7; Hill, 2005; Jorquera, 20II). Lo que aparece es la «estrategia del agandallamiento» (Camus, 20I4, p. I2): falla sistemáticamente el control social formal e informal y se imponen otras normas por la vía de la costumbre y la ley del más fuerte. Con Janowitz (1975, pp. 82, 87, cit. en Sampson y Raudenbush, 1999, pp. 610-613), entendemos el control social como «la capacidad de una unidad social para regularse a sí misma de acuerdo con 
principios deseados para realizar metas colectivas, en oposición a metas forzadas o impuestas». Los indicadores del control social informal que hablan de agandallamiento también están referidos a los espacios públicos (como en la investigación de Sampson y Raudenbush, 1999): música con volumen alto y a todas horas, los carros se estacionan en lugares indebidos, los canceles eléctricos se abren por la fuerza, etc. (Camus, 20I4).

\section{POSICIONAMIENTO E IDENTIDAD: CRUCE DE ANÁLISIS DE CONGLOMERADOS Y DE REPERTORIOS INTERPRETATIVOS}

\section{MÉTODO PARA LA PRODUCCIÓN Y ANÁLISIS DE LOS DATOS DE LA INVESTIGACIÓN}

El cuerpo de datos para este análisis procede de una investigación previa ${ }^{6}$ sobre la relación entre el sentimiento de inseguridad, la eficacia colectiva y la estigmatización territorial. Siguiendo a Kessler (2009, cita a Ferraro, 1995), se entiende el sentimiento de inseguridad como la experiencia emocional (de miedo, enojo, frustración, etc.) impelida por la percepción en el entorno de riesgos de sufrir daños personales o patrimoniales; puede estar asociada a juicios sobre la importancia de la inseguridad como problema público y a la preferencia por la adopción de medidas para reducirla. Retomando la definición de Sampson y Raudenbush (1999), se entiende por eficacia colectiva a la cercanía social y en expectativas compartidas para poder actuar colectivamente en apoyo del logro de metas que son comunes al colectivo mismo. Hablamos de estigmatización territorial cuando nos referimos «a ese proceso por el cual un determinado espacio queda reducido a ciertos atributos negativos, que aparecen magnificados, estereotipados, produciendo como resultado una devaluación o desacreditación social del mismo» (Kessler y Dimarco, 2013, p. 225).

\footnotetext{
${ }^{6}$ Agradezco el apoyo del conacyt en la modalidad de beca para los estudios de posgrado. Algunos fragmentos de este artículo han sido retomados de la tesis doctoral (Foust, 2015) y de una ponencia (Foust, 20I6).
} 
Como han señalado muchos autores (Caldeira, I996 y 2000; Jackson, Farrall y Gray, 2007; Jorquera, 20II; Hill, 2005; Kessler, 2009; Low, 200I; Tulloch, 2003; Wacquant, 200Ia y b, 2007 y 20II), la exploración amplia de significaciones en torno al sentimiento de inseguridad (miedo al crimen es la expresión más usual) abre la cortina a una ventana más amplia de discursos y prácticas en relación con procesos de diferenciación y estigmatización social. En el intento por asomarnos a esta ventana, se diseñó una aproximación de método mixto (Creswell, 2003) y se realizó trabajo de campo en un fraccionamiento de clase media-baja en Tlajomulco de Zúniga, Jalisco, entre 2012 y 20I4, que consistió en encuesta a hogares, entrevistas semiestructuradas y observación participante y no-participante.

Para el análisis que se presenta en este texto, se trabajó a partir de una submuestra de una muestra original de 135 hogares (49 de Valle del Ascenso y 86 de un fraccionamiento vecino, muy populoso, de hogares de bajos ingresos). El diseño de la muestra fue aleatorio simple, la unidad de selección fue la vivienda y la unidad de observación fue el hogar, aunque no se hizo un muestreo al interior del hogar, sino que se entrevistó a la persona que atendió la puerta y quiso responder al cuestionario (siempre y cuando tuviera al menos is años de edad). El marco muestral fue el conocimiento del número de cotos. Se tomó como punto de referencia una esquina y se contó el número de viviendas equivalente al cociente del número aproximado de viviendas entre el tamaño de muestra.

Se aplicó un cuestionario a 49 personas ( 23 hombres y 26 mujeres) que fueron localizadas en sus hogares a distintas horas del día. Desde el principio, se pretendió activamente que el cuestionario fuera un pretexto para una entrevista más amplia, que se logró con alrededor de una veintena de participantes. Los cuestionarios y entrevistas se transcribieron y luego fueron analizados - por separado y triangulándolos- con varias técnicas de análisis estadístico (véase Tabachnick y Fidell, 1996) y siguiendo, en parte, los procedimientos y pistas de validación de la teoría fundamentada (Corbin y Strauss, 1990) y del posicionamiento (Bamberg, I997; Davies y Harré, I999; Korobov, 200I).

De forma similar a como lo concibe Tulloch (2003, pp. 474-475), entendemos que la identidad - «el conjunto de significados que las personas aplican a sí mismos como miembros de diferentes grupos [...], ocupantes 
de diferentes roles [...]» (Burke y Stets, 2009, cit. por Stets, 20I2, p. 327)- se produce discursivamente en el contexto de una situación particular de interacción con la persona que investiga, posicionándose el(la) entrevistado(a) ante una audiencia imaginada, pero, también, como manifestación de una configuración dinámica relativamente persistente en el entramado complejo en el que nos desarrollamos como personas (véase también Bamberg, 1997; Davies y Harré, I999; Korobov, 200I). Tulloch (2003) propone un modo de aproximarse a esta construcción discursiva dinámica de la identidad, interpretando las respuestas a los cuestionarios como estrategias de posicionamiento de los participantes: analizando vis-à-vis las consistencias e inconsistencias en las respuestas de los participantes mediante el análisis de conglomerados (cluster analysis) y de «repertorios interpretativos»: «sistemas de términos recurrentes utilizados para caracterizar y evaluar acciones, eventos y otros fenómenos» (Potter y Wetherell, 1987, p. I49, cit. en Wooffitt, 2005, p. 35; cf. Wetherell, 1998, p. 400, cit. en Tulloch, 2003, p. 467).

En un primer momento de análisis, se hizo una (re)lectura de los testimonios recogidos en las entrevistas para tratar de identificar estos repertorios interpretativos. Los testimonios de los(as) entrevistados(as) giran en torno a cuatro «nodos de significado» (Creswell, 2007, p. 6I): (I) las grietas de la integración social hacia arriba y las estrategias de agandallamiento; (2) desilusión y frustración en torno al proyecto aspiracional; (3) ansiedad por el estatus, y (4) «sueños de fuga».7 Estos tres nodos se articulan entre sí en los posicionamientos de los entrevistados: algunos -la mayoría- se escuchan desilusionados y frustrados en torno al proyecto anunciado por las inmobiliarias que promueven estos desarrollos, tal vez incluso agotados por intentar materializar la «comunidad de semejantes» (Calonge, 2013, pp. I50 y 153) y el lugar seguro, cómodo, autogestivo que querían comprar.

Se buscaron las variables que pudieran estar más asociadas a estas narrativas para agrupar a los entrevistados(as) en conglomerados. Y luego se hizo una segunda relectura de los testimonios. Las variables utilizadas para el análisis fueron:

7 Para usar el sugerente título de una historia escrita por Stephen King (1982), hecha película después (Darabont, 1994). 
- Edad de la persona entrevistada.

- Tamańo del hogar.

- Ingreso per cápita mensual. Se usó un proxy: el ingreso promedio correspondiente al máximo grado escolar completado fue imputado al perceptor principal; luego se dividió dicho ingreso entre el número de personas en el hogar.

- Años de residencia en el fraccionamiento.

- Percepción de respeto entre vecinos. Medición de nivel ordinal (posteriormente se construyó un índice de nivel intervalo) de percepción de respeto a la persona y las cosas entre vecinos, usando esta pregunta: "¿Qué tan de acuerdo o en desacuerdo está con esta frase?: «En esta colonia hay que estar alerta de los vecinos o se aprovechan de uno».8

- Percepción de eficacia colectiva. Siguiendo a Sampson y Raudenbush (1999), el índice de eficacia colectiva se construyó integrando (sumando) los índices de cohesión social y de expectativas de control social informal en los espacios públicos; a su vez, la cohesión social se basó en una medición ordinal de la percepción de unidad, cercanía y afinidad en valores de los vecinos; la expectativa de control social informal se estimó con base en una medición ordinal de la percepción de un consenso (y apego a él) en torno a principios o políticas para el cuidado en común de los espacios públicos.

- Percepción de desorden. Se realizó una medición (ordinal que luego se convirtió a un índice de nivel de intervalo) de la percepción de señales físicas o sociales de daño a la propiedad o de agresión potencial o actual en espacios públicos. ${ }^{9}$

- Percepción de apoyo mutuo entre vecinos. ${ }^{10}$

${ }^{8}$ La pregunta que fue incluida en el cuestionario fue retomada de estudios previos (véanse Conkin, 1971, y Sampson y Raudenbush, 1999).

${ }^{9}$ Las preguntas incluidas en el cuestionario fueron tomadas de estudios previos (véanse Jackson, Farrall y Gray, 2007; Sampson y Raudenbush, 1999; Skogan, 1986).

${ }^{\text {ro }}$ Muy similares a las preguntas incluidas en el módulo de redes sociales de la Encuesta Nacional de Ingresos y Gastos de los Hogares. 
- Percepción del afecto por la comunidad y sentido de pertenencia. Medición de la percepción de la satisfacción con la comunidad como lugar para vivir y para ver crecer a los hijos; deseos de mudarse. ${ }^{\text {II }}$

- Preocupación por la precariedad económica en el hogar. Medición de la frecuencia e intensidad de las emociones ${ }^{12}$ frente a las manifestaciones de precariedad económica; rango de tiempo de referencia: I2 meses.

Se realizaron siete ejercicios explorando en la base de datos de la encuesta en hogares anteriormente referida para identificar conglomerados. Se empleó una técnica de conglomerados no jerárquicos (k-medias) y una de conglomerados jerárquicos (Ward) para validar la estabilidad de estos conglomerados. Los resultados de ambas técnicas fueron similares en un rango de 75 a Ioo por ciento. Confirmado por el análisis de función discriminante que se presenta posteriormente, este es el análisis de conglomerados que mejor agrupó los datos (Tabla I y 2):

Tabla I. Conglomerados (media y desviación estándar en el centro de cada conglomerado)

\begin{tabular}{|c|c|c|c|}
\hline & $\begin{array}{l}\mathbf{I} \\
(\mathbf{N}=\mathbf{I I})\end{array}$ & $\begin{array}{l}2 \\
(N=27)\end{array}$ & $\begin{array}{l}3 \\
(N=I I)\end{array}$ \\
\hline Años de residencia & $\begin{array}{c}5.4 \\
(3.32)\end{array}$ & $\begin{array}{l}2.9 \\
(2) \\
\end{array}$ & $\begin{array}{c}5.5 \\
(2.2) \\
\end{array}$ \\
\hline Eficacia colectiva & $\begin{array}{c}54.4 \mathrm{I} \\
(\mathrm{I} 8.64)\end{array}$ & $\begin{array}{c}38.12 \\
(\mathrm{I} 0.53) \\
\end{array}$ & $\begin{array}{c}50.01 \\
(\mathrm{I} 3.73) \\
\end{array}$ \\
\hline Apoyo mutuo & $\begin{array}{c}64.56 \\
(30.2 I) \\
\end{array}$ & $\begin{array}{l}32.35 \\
(\mathrm{I} 2.4) \\
\end{array}$ & $\begin{array}{l}90.90 \\
(15.56) \\
\end{array}$ \\
\hline Afecto por la comunidad y sentido de pertenencia & $\begin{array}{l}97.8 \mathrm{I} \\
(7.24)\end{array}$ & $\begin{array}{l}35.20 \\
\text { (II.4) }\end{array}$ & $\begin{array}{c}\text { 4I.I4 } \\
\text { (I2.69) }\end{array}$ \\
\hline
\end{tabular}

Tabla 2. Prueba de igualdad de medias de los grupos

\begin{tabular}{lccc}
\hline & Wilk's Lambda & F & Sig. \\
\hline Años de residencia & 0.775 & 6.675 & .003 \\
\hline Eficacia colectiva & 0.767 & 7.004 & .002 \\
\hline
\end{tabular}

${ }^{\text {II }}$ Se usaron preguntas retomadas de estudios previos (véanse Conklin, 197I; Fidel, Di Tomasso y Farías, 2008; Sampson y Raudenbush, I999).

${ }^{12}$ Con base en Jackson (20IO). 


\begin{tabular}{llll}
\hline Apoyo mutuo & $0.35 \mathrm{I}$ & $42.56 \mathrm{I}$ & .000 \\
\hline Afecto por la comunidad y sentido de pertenencia & 0.148 & $132.89 \mathrm{I}$ & .000 \\
\hline
\end{tabular}

Se hizo un análisis de función discriminante (Gráfica I) para confirmar que la combinación de variables identificada pudiera ofrecer una clasificación en la cual los grupos no se traslaparan y las dimensiones fueran estadísticamente significativas. Los resultados se presentan en la tabla 3:

Tabla 3. Correlaciones de las variables predictivas con las funciones discriminantes

\begin{tabular}{lcc}
\hline & Función \\
\hline & $\mathrm{I}$ & 2 \\
\hline Afecto por la comunidad y sentido de pertenencia & .953 & -.162 \\
\hline Apoyo mutuo & .237 & .885 \\
\hline Años de residencia & .154 & $.27 \mathrm{I}$ \\
\hline Eficacia colectiva & .189 & .205 \\
\hline Porcentaje de varianza por función & 76.8 & 23.2 \\
\hline
\end{tabular}

Gráfica I. Funciones discriminantes, grupos identificados y centroides de los grupos.

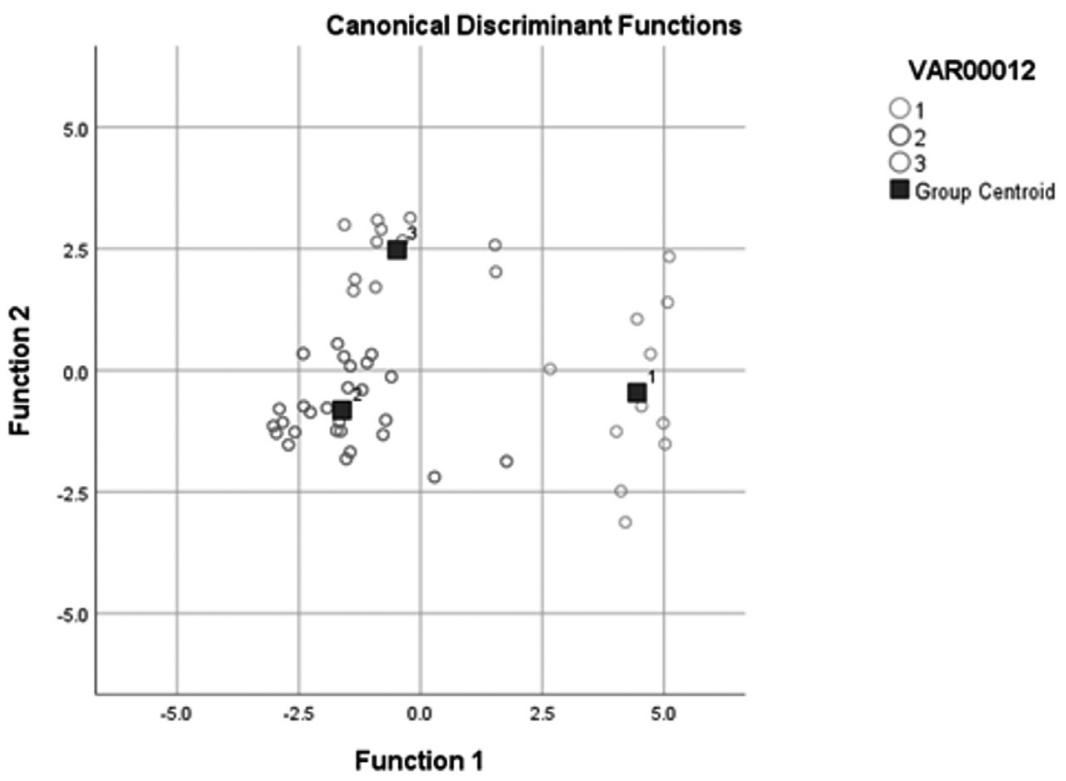


Como podemos observar, se pudieron identificar dos funciones discriminantes significativas, que dan cuenta del $76.8 \%$ y $23.2 \%$ de la varianza, respectivamente. La función 1 tiene una correlación de 0.953 con la variable afecto por la comunidad y sentido de pertenencia; la función 2, de 0.885 con la variable apoyo mutuo. La gráfica 2 muestra los tres conglomerados en un plano cartesiano: el grupo mayoritario (número 2) tiene valores bajos en ambas funciones, por eso está ubicado abajo y a la izquierda; el grupo 3 tiene valores más altos en la variable de apoyo mutuo, pero bajos en la otra variable. El grupo 1 tiene valores muy altos en la variable de afecto por la comunidad y sentido de pertenencia (por eso está en el extremo derecho de la gráfica), pero se dispersa en relación con la variable de apoyo mutuo (su desviación estándar representa la mitad de su media). Esto quiere decir que algunos miembros de este grupo no tienen la expectativa de ser apoyados por sus vecinos, pero aun asi preferirian quedarse en este fraccionamiento. Este aspecto es muy relevante para el análisis, como comentaremos más adelante.

Otras características de estos grupos pueden consultarse en las tablas incluidas en el Anexo. (Tabla $\mathrm{A}_{1}, \mathrm{~A}_{2} \mathrm{Y} \mathrm{A}_{3}$ ).

\section{POSICIONAMIENTO E IDENTIDAD DE LOS HABITANTES DE VALLE DEL ASCENSO}

El grupo mayoritario (con el número 2 en la Gráfica I) está conformado por $55 \%$ de los hogares; dos tercios de ellos son no pobres y no vulnerables (en contraste con poco más de un tercio al interior del grupo i en la gráfica). A los entrevistados de este grupo los une la percepción de que el fraccionamiento está igual o peor que antes (véase la tabla A2 del Anexo): acusan a sus vecinos de hacer fiestas ruidosas sin respetar el sueño de los demás, de no levantar las heces de sus perros, de dejar que sus hijos adolescentes maltraten los parques o consuman marihuana (estas percepciones se reflejan en los valores bajos reportados en las variables afecto por la comunidad y sentido de pertenencia, de apoyo mutuo y de eficacia colectiva en la tabla i).

¿En esta colonia lo respetan a uno? [...] Un vecino instaló un asador en el área común $[. .$.$] Hay cacas de perro.$ 
Los problemas de la colonia crean rencillas entre los vecinos y falta de acuerdos. No todos pagan las cuotas. Quieren que todo esté bien, pero sin pagar [...] Esta colonia va en decadencia: escuincles de secundaria echando alcohol... ¡Está cabrón!

(Hombre, casado, 38 años, puesto gerencial medio).

Frisch, González y Steinhardt (20I7) hablan de «fisuras» en estos microsistemas sociales; y Svampa (20I4) se refiere a las dificultades en la «integración hacia arriba». En algunas de las personas entrevistadas escuchamos estos lamentos por la falta de cierre del sistema social:

En la Plaza $(\mathrm{X})$ va gente de todos lados [...] no sabe uno.

(Mujer, 42 años, ama de casa; énfasis nuestro).

«Va gente de todos lados», es decir: también de los fraccionamientos vecinos, más populosos, estigmatizados como violentos e inseguros con hogares de ingresos bajos, etcétera.

Otro entrevistado alude a normas de comportamiento que suponen respeto de los vecinos y que, de acuerdo con su perspectiva, deberían ser observadas: no poner música a un volumen demasiado alto; no poner música que termina siendo una apología del delito; no usar las casas-habitación para instalar negocios que producen molestias para los demás:

Falta cultura en cuanto a respetar el entorno. [Uno] no puede andar poniendo música alta -inarco-corridos!- [...] Se suben a las camionetas y se amanecen [...] Se están hasta las tres o cuatro de la madrugada. Y la gente tiene que ir a trabajar [...] Otro vecino tiene un taller mecánico en su casa, y eso produce ruidos y olores...

(Hombre, jubilado, 72 años).

Temen que el fraccionamiento siga en picada y «se convierta en X» (mencionan un asentamiento vecino que es como un sinónimo del estigma de lo vio- 
lento, inseguro, sobrepoblado, conflictivo, etc.). Más que lamentarse por la caída de la plusvalía, se les escucha desesperanzados, resignados, derrotados.

Tengo mi perrita y le doy su vuelta. Llevo mi bolsa y me doy cuenta que hay muchas cacas, y las personas salen con sus perros y no llevan bolsita. Antes los confrontaba más, les llamaba la atención. Ahora lo dejo pasar para no meterme en lios [...] Me siento decepcionado.

(Hombre, 55 años, casado, profesor universitario; énfasis nuestro).

Al principio yo regaba el parque y todo, pero ya no [...] Les llama uno la atención si maltratan algo y hasta se burlan [...] Llamé [a la vigilancia, de la administración de colonos] para quejarme de unos vecinos que no dejaban dormir y les dijeron a mis vecinos quién había llamado: ¡me los echaron encima! ¡Qué [in]discreción! Vivo aqui porque ya tenemos la casa; si no, no. Al principio hasta me sentía orgulloso, pero con la clase de arañas que tenemos de vecinos [...] se te cae el corazón. (Hombre, 73 años, casado; énfasis nuestro).

En estos dos testimonios se puede observar el cambio de actitud y de sentimiento: en un caso, apelando a ciertas normas de convivencia, el entrevistado interpelaba a sus vecinos; en otro caso, incluso se sentía orgulloso (de vivir en este fraccionamiento, puede suponerse); en ambos casos, reportan ahora decepción, tristeza («se te cae el corazón»). También se ha dejado de lado la confrontación, "para no meterse en líos», pues las otras personas no se remiten a la norma, sino a la disputa por la fuerza, situación a la que se niegan estos entrevistados. Uno de ellos se refiere a sus vecinos como «esta clase de arañas».

Hochschild (1979) habla del trabajo emocional que hacen las personas para lograr un ajuste entre su experiencia emocional, las reglas de esta experiencia y el entorno social en el que se presenta. Distingue tres tipos de técnicas de trabajo emocional: (a) la cognitiva, que se distinguen por los «intentos de recodificiación de una situación»; (b) la corporal, en donde se trata de buscar un ajuste en el componente físico-somático de la emoción; (c) la expresiva, en donde se trabaja la expresión, la manifestación de lo que 
se está sintiendo (ibíd., p. 562). En ambos casos anteriores también puede entreverse el «trabajo emocional» que están haciendo los sujetos para sobrellevar una situación en la que se sienten resignados. "Vivo aquí porque ya tenemos casa; si no, no", dice el hombre mayor.

El grupo número 3 (22.5 por ciento de los hogares) se distingue por tener una percepción de mayor apoyo mutuo en el fraccionamiento (tabla I). Una de las entrevistadas, en un tono sutil, matizaba las opiniones de sus vecinos con las suyas, y destacaba los aspectos que ella percibe como positivos en el fraccionamiento:

A mí se me hace muy seguro; vivo muy segura aquí, pero los vecinos dicen que se ve en los parques: chavos que están drogándose. Yo tengo buena relación con los vecinos. Uno que otro no deja bien cerrada la bolsa de la basura o no quieren pagar las cuotas de mantenimiento del coto, o que usan palabras altisonantes o que no les importa pelearse a gritos. (Mujer casada, 39 años, profesionista).

La entrevista se realizó en uno de los parques principales, en donde está la oficina de la Administración de la Asociación de Colonos. Tenía mucha vida (alrededor de las siete y media de una tarde de abril): había niños jugando con sus papás o sus mamás, clase de hawaiano, clase de pintura en cerámica para niños, adultos acordando cosas o tratando asuntos con los administradores de la Asociación de Colonos. Ella comentó: «este parque se siente vivo, pero otros no se sienten así».

Finalmente, el grupo número I está conformado por $22.5 \%$ de la muestra. Lo que aglomera a estos hogares es una percepción más optimista del fraccionamiento respecto al grupo mayoritario: no comparten con la mayoría que el fraccionamiento está peor que antes y no tienen deseos de mudarse ${ }^{13}$ (véanse las Tablas Ar, A2 Y A3 del Anexo).

$\mathrm{Al}$ interior de este conglomerado se pueden distinguir dos subgrupos. El primer subgrupo está conformado por varios hogares en ascenso socioe-

${ }^{13}$ Todos los hogares de este grupo (II) se encontraron en el punto extremo de una escala de Likert que medía el deseo de mudarse o no mudarse; la diferencia es estadísticamente significativa respecto a los otros grupos (prueba Kruskal-Wallis). 
conómico. Al momento de la encuesta registraban condiciones de pobreza moderada o con vulnerabilidad por ingresos o por carencias. ${ }^{14}$ Reportaban que su situación socioeconómica había mejorado, si bien sus ingresos siguen siendo bajos (alrededor de I5 mil pesos mensuales por hogar; en hogares de 4.8 miembros, en promedio). Uno de estos hogares es el de Laura' ${ }^{15}$ (de 40 años, nueve rentando en este lugar), se mudó junto con su familia, huyendo de la inseguridad. Antes vivía cerca de una de las zonas con mayor índice delictivo en la ciudad. Espera que Valle del Ascenso siga así de seguro. «Aquí es una vida más familiar. Allá había muchos cholos. En [X colonia] hay por montones», dice. Piensa que la colonia estará mejor en términos de respeto, confianza interpersonal, etc., "pues ha habido cambios y se supone que es para mejorar. Alfaro hizo mucho por Tlajomulco, ahora a ver cómo nos va, también con el cambio de Administración de Colonos». Su percepción es que «diario [siempre] así está, ¡mire!». Había varios niños jugando en el parque, que además estaba limpio. «Puede ver a mujeres con escobas y recogedor, haciendo limpieza. A muchachitos a las nueve o diez de la noche platicando o con sus celulares [...] Es por el mantenimiento que se paga».

Mientras otros ven parques maltratados y jóvenes consumiendo drogas, Laura ve jardines limpios y adolescentes que pueden convivir por las noches. «iMire!», exclama, como apelando a la atención del entrevistador para que registre esta visión.

El otro subgrupo está compuesto por hogares con ingresos medios (respecto al conjunto de la muestra), sin deseos de mudarse del fraccionamiento. No comparten que el fraccionamiento está peor que antes, pero tampoco apuestan a que vaya a mejorar; en todo caso, se mudarían a algo mejor. Este subgrupo tiene una más alta percepción de que sus vecinos se están organizando para realizar acciones a favor del bien común.

Carlos, un profesor pensionado, vive con su esposa, también pensionada. Es el comisario de su coto, encargado de estar atento a ver quién entra al coto. Dice que cuando alguien extraño entra, «lo invita a salir», como a los Testigos

${ }^{14}$ Recordemos que estamos usando una aproximación al método oficial de medición de la pobreza de CONEval.

is Los nombres han sido cambiados para preservar la confidencialidad de las personas entrevistadas. También se han evitado otros datos que pudieran conducir a identificarlos. 
de Jehová, «que no tienen nada que hacer aquí». Él y su esposa afirmaron que hace poco hubo patrullas dentro del fraccionamiento, pues hubo un robo de auto. "No había ocurrido antes y ya empezó». También dicen que hace poco se metieron a robar en la casa de su hijo, quien también vive en fraccionamiento. "Gente de fuera que rentó toldos».

Carlos ejemplifica la actitud de algunos entrevistados de estar activamente organizados para la defensa de su $\operatorname{coto}^{16}$ (y, en el fondo, de proyecto del fraccionamiento): vigila, controla el acceso. Si hay actos de delincuencia, los habrán perpetrado «los de afuera» porque «antes no había ocurrido».

Además de estos tres grandes conglomerados, al triangular los diferentes ejercicios de análisis estadístico con los discursos de las personas entrevistadas, se identificaron algunos subgrupos con características específicas que resulta pertinente destacar. Uno de estos subgrupos está constituido por matrimonios jóvenes, sin hijos o con solo un hijo(a). Con ingresos relativamente más altos que el resto ${ }^{17}$ (perciben 35 mil pesos mensuales por hogar, en promedio); no manifiestan estar preocupados por el desempleo o por su situación económica. Otro subgrupo lo forman personas de la tercera edad (de 55 a 73 años) - a quienes hemos escuchado en los párrafos anteriores-con un nivel relativamente alto de preocupación por la vulnerabilidad de su situación económica ${ }^{\mathrm{I} 8} \mathrm{y}$ una percepción de deterioro progresivo del fraccionamiento. ${ }^{19}$

Finalmente, lo que tal vez llame más la atención al realizar este ejercicio de análisis es la detección de algunos hogares que se encuentran en una suerte de intersección social, en cierto grado de ambivalencia o ambigüedad, pues en algunos de los ejercicios de análisis de conglomerados quedaban clasificados en un grupo y en otros casos quedan clasificados en otro. Estos hogares parecen ser los más representativos de la tensión que generan la ansiedad de estatus y el deseo de integrarse hacia arriba.

\footnotetext{
${ }^{16}$ Un pleonasmo, si seguimos la definición de coto ya ofrecida antes.

${ }^{17}$ Se realizó una prueba Kruskal-Wallis para determinar que la diferencia de ingresos entre grupos era estadísticamente significativa (nivel de significancia de 0.05).

${ }^{\mathrm{I} 8}$ Diferencia estadísticamente significativa en un nivel de significancia de 0.06 (prueba de Kruskal-Wallis).

${ }^{19}$ Diferencia estadísticamente significativa en un nivel de significancia de 0.098 (prueba de Kruskal-Wallis).
} 
Rosaura, de I9 años, estudia en la universidad. Antes vivía en otra colonia periférica, pero de hogares con menores ingresos. A sus padres les gustó Valle del Ascenso por los jardines y el ambiente tranquilo. Adquirieron crédito aquí, pero ya no pueden pagarlo. Ahora forman parte de El Barzón. ${ }^{I}$ Cuando se le pregunta si le gustaría que sus hijos crecieran en este fraccionamiento, responde: «No, me gustaría algo más fresón». ¿Qué es más fresón?, se le pregunta: «con más privacidad, como tipo residencial»; suponemos que se refiere a un fraccionamiento de clase alta. Meses después de la entrevista, ella y su familia tuvieron que mudarse a la casa en la que vivían antes.

Álex (33 años) es comerciante y antes tenía otro trabajo. "Me quedo en este fraccionamiento para que mis hijos suban un escalón y crezcan con gente de otra clase», comenta (el énfasis es nuestro). Su esposa acababa de ser operada en el Hospital Civil de Guadalajara, de emergencia por apendicitis. La operación fue costeada con un ahorro que la familia tenía para eso precisamente. La preocupación principal de Álex es quedarse sin trabajo o tener menos ingresos. "Yo creo que por eso me acaba de dar gripa, por la preocupación».

Alex se refiere a su situación actual como una mejoría en su situación socioeconómica. En particular, se remite al proceso de socialización de sus hijos: «en el escalón de arriba [...] con gente de otra clase», aunque la preocupación por sostener el costo de vivir en «ese escalón» parece estarle cobrando factura. También comenta:

Cuando llegué, estaba mejor. Ahora está más vandalizado: robos de carro, de casa [...] Cuando llegué no se oía tanto. Hay pedazos bien cuidados y otros en donde se juntan a estarse drogando [...] Ya se están destapando. Ya les valió. Se está viendo como normal. (énfasis nuestro).

Quisiera mudarse, pero «para mejorar». Algo similar expresa Juanita, quien tiene seis años viviendo en casa propia. Su esposo es agente de ventas y dos de sus hijos también trabajan (uno de cajero y otro en telemarketing).

I El Barzón es una organización de personas que se vieron afectadas por el alza súbita de las tasas de interés durante la crisis hipotecaria de 1994-95. Su lema es «Debo, no niego. Pago lo justo». 
Me gustaría vivir en un ranchito, de esos con casas grandes que huelen rico, a viejas, como en Tapalpa [...] Si me voy a vivir a otra casa igual, pues mejor me quedo aqui.

(Mujer, casada, 40 años).

En la voz de otra entrevistada se nota todavía más el tono de contradicción que quiere salvarse: en su coto automatizaron el cancel hace un par de años. Algunos se opusieron al cancel eléctrico por el costo. Ella los critica porque «tienen ideas como de barrio». «¿Para qué pones cancel?, si no vivimos en Puerta de Hierro [...] Yo no tengo carro', me decían esas vecinas», señala. Adriana contradice su propio discurso, pues trabaja en su casa como estilista, violando las reglas que protegen el concepto de fraccionamiento con espacios para negocios separados de las casas. Ella está consciente de esto y se justifica de cara al modo como ha posicionado a su vecina (quien se había opuesto al cancel y, según Adriana, generó división):

Ha bajado mi clientela porque no abren y yo a veces no puedo abrir, pero no puedo exigir nada porque esta es una casa [...] Sí me han hecho bronca [...] la vecina. Se dijo a los del municipio [ayuntamiento] y la asociación [de colonos] que no tenía permiso para poner una bardita, y que no era conforme al estilo de la construcción. Cuando llegaron con ella, me los echó.

Ella tiende la ropa en el cancel. Y se ve mal su tenderete de vecindad. Yo no interrumpo áreas verdes, ni atiendo a mis clientes afuera; yo voy a abrirles (el énfasis es nuestro).

En estos testimonios notamos las tensiones entre el proyecto de ascenso y la realidad socioeconómica de los entrevistados. En la voz de Adriana además es notorio el esfuerzo por salvar estas contradicciones y justificar la crítica a sus vecinas, que siguen «teniendo ideas como de vecindad». 


\section{DISCUSIÓN Y CONCLUSIONES}

Al exponer el dinamismo de discriminación y estratificación social que va configurando a una clase como el estándar a imitar, Veblen (2009/1899: introducción y capítulo I) afirma que los hombres -especialmente los más fuertes o hábiles para la caza y la guerra- se van apropiando de un rol depredador, y van limitando a las mujeres a las tareas más rutinarias e industriosas de la procuración de la subsistencia. Se van distinguiendo dos tipos de actividades, aquellas caracterizadas como hazañas o proezas, y otras menos glamourosas, pero indispensables para la reproducción material del grupo. Con el surgimiento de la propiedad privada, las disputas por la fuerza no desaparecen, pero la hazaña consiste ahora en la acumulación de propiedad o, al menos, de un estándar material promedio.

Aquellos miembros de la comunidad que quedan por debajo de este grado algo indefinido de proeza o de propiedad normal sufren en la estima de los demás; y, consecuentemente, sufren también en su autoestima, dado que la base usual del auto-respecto es el respeto de acuerdo con los propios vecinos. [...] Y una vez que ha alcanzado lo que podría llamarse el estándar pecuniario normal para su comunidad o su clase, esta crónica insatisfacción dará lugar a una tensión incesante para poderse colocar por encima $-\mathrm{y}$ cada vez más arriba- del estándar promedio. (Veblen, 2009/1899, pp. 25-26).

Como hemos notado (en convergencia con Frisch, González y Steindhartd, 20I7), al igual que otros fraccionamientos cerrados de clase media, Valle del Ascenso tiene amplia heterogeneidad socioeconómica. Esta puede leerse desde el filtro vebleniano del «estándar pecuniario normal para su comunidad o clase». En nuestro caso, los hogares del grupo I son los que tienen menores ingresos (si bien la diferencia no es estadísticamente significativa). Las voces de varias personas de este grupo, y de otro subgrupo identificado, dan para pensar que hay mayor o menor «ansiedad por el estatus». También tenemos elementos para pensarlo así al revisar las variables significativas para la clasificación en grupos: los valores de la variable apoyo mutuo se dispersan 
en el caso del grupo I, mientras que son el grupo con los registros más altos en la variable de afecto comunitario y sentido de pertenencia. Esto quiere decir que algunos hogares no perciben que en su fraccionamiento la gente se apoye entre sí (o sea, no se sienten apoyados), pero, a pesar de esto, no quieren mudarse. Hay un claro contraste con el grupo 3: ellos perciben que en el fraccionamiento se apoyan entre sí, pero preferirían mudarse y que sus hijos no crecieran ahí. El grupo mayoritario -el 2- de plano tiene sueños de fuga, sus miembros se sienten desilusionados, fatigados, con un proyecto social frustrado.

Estas tensiones y contradicciones parecen estar siendo experimentadas con mayor intensidad por el subgrupo que caracterizamos como estando en la intersección de este microcosmos social. Así lo muestra el esfuerzo por posicionarse de cara al entrevistador y, por tanto, ante un auditorio imaginario (véase Davies y Harré, 1999) con una identidad que busca ser verificada por el otro, a pesar de las incoherencias (véase Stets, 20I2).

En su texto clásico, Jane Jacobs advirtió que «el eslabón clave de un barrio bajo perpetuo es que demasiada gente se marcha de él demasiado rápido y, mientras tanto, sueñan con hacerlo» (201I/196II, p. 308). Aunque no es un barrio bajo, en Valle del Ascenso unos sueñan con mudarse (los grupos 2 y 3) y otros sueñan con ser integrados, reconocidos como semejantes (el grupo I) por aquellos que los ven como gandallas que maltratan los espacios públicos y no respetan las normas implícitas de convivencia. Mientras no se mudan, no se integran y no se reconocen entre sí, tampoco se perciben como capaces de lograr metas comunes: perciben que su nivel de eficacia colectiva es débil (oscila entre 38/IO0 y 54/IOO).

Estos procesos de identidades estresadas llaman nuestra atención hacia la violencia cotidiana y normalizada que constituye la neoestamentalización de la metrópolis tapatía y, muy probablemente, de otras metrópolis latinoamericanas.

\section{REFERENCIAS}

Bamberg, M.G.W. (1997). Positioning Between Structure and Performance. Journal of Narrative and Life History, 7(I-4), 335-342. 
Calonge Reíllo, F. (2013). Los sentidos de la ciudad. Sobre cómo mujeres y hombres ordenan sus espacios vitales. Universidad de Guadalajara.

Bourdieu, P. (1996/1979). Distinction: a Social of the JUDGMENT of Taste. Harvard University Press.

Bronwyn, D. y Harre, R. (I999). Posicionamiento: la producción discursiva de la identidad. Sociológica, I4(39), 2I5-239.

Bruin, J. (2006). Newtest: Command to Compute New Test. UCla: Statistical Consulting Group. <https://stats.idre.ucla.edu/spss/ output/discriminant-analysis/ $>$.

Cabrales, L.F. y Canosa, Z.E. (200I). Segregación residencial y fragmentación urbana: los fraccionamientos cerrados en Guadalajara. Espiral. Estudios sobre Estado y Sociedad, XII(20), 223-253.

Caldeira, T. (1996). Fortified Enclaves: The New Urban Segregation. Public Culture, 8(2), 303-328.

Caldeira, T. (2000). City of Walls. Crime, segregation and Citizenship in São Paulo. University of California Press.

Camus, B.M. (20I4). Sobre los condominios wannabe o del coto a las aspiraciones. Guadalajara: Centro de Estudios de Género, Universidad de Guadalajara (texto no publicado).

Camus, B.M. (2015). Vivir en el coto. Fraccionamientos cerrados, mujeres y colonialidad. Universidad de Guadalajara.

Camus, B.M. (2018, agosto 30). Habitar el privilegio. Centro para la Cultura Arquitectónica y Urbana. Conferencia pronunciada en el ciclo de otońo de los Jueves de CCaU. Guadalajara, Jalisco. $<$ https://vimeo.com/291509796>.

Consejo Nacional de Evaluación de la Política de Desarrollo Social (CoNEVAL) (2009). Metodología para la medición multidimensional de la pobreza en México. CONEval.

Conklin, J. (I97I). Dimensions of Community Response to the Crime Problem. Social Problems, I8(3), 373-385.

Corbin, J. y Strauss, A. (1990). Grounded Theory Research: Procedures, Canons and Evaluative Criteria. Zeitschriftfür Soziologie, I9(6), $418-427$. 
Coulomb, R. (2007). El Estado mexicano y el derecho a la vivienda. Alternativas de política. En José Luis Calva (coord.), Agenda para el desarrollo. Derechos y politicas sociales (vol.I2, 255-270). UNAM.

Creswell, J. W. (2003). Research Design. Qualitative, Quantitative and Mixed Methods. Sage Publications.

Creswell, J.W. (2007). Qualitative Inquiry \& Research Design. Choosing Among Five Approaches (second edition). Sage Publications.

Darabont, F. (1994). The Shawshank Redemption. Columbia Pictures. Castle Rock Entertainment.

Fidel, C.H., Di Tomaso, R. y Farías, C. (2008). Territorio, condiciones de vida y exclusión: el Partido de Quilmes (Provincia de Buenos Aires, Argentina). Consejo Latinoamericano de Ciencias Sociales.

Foust Rodríguez, D. (2015). Sentimiento de inseguridad, estigmatización territorial y eficacia colectiva en dos colonias de la periferia metropolitana de Guadalajara. Tesis doctoral no publicada. Universidad de Guadalajara.

Foust Rodríguez, D. (2016). Sentimiento de inseguridad, estigmatización territorial y eficacia colectiva en dos fraccionamientos de la periferia metropolitana de Guadalajara. Ponencia presentada en el $V$ Coloquio de investigación Las emociones en el marco de las ciencias sociales: perspectivas interdisciplinarias, realizado en ITEso los días 22 y 23 de septiembre de 2016.

Frisch, M., Agustina, E., Gabriel González, M. y Steinhardt, L. (diciembre de 20I7). Procesos de diferenciación y estructuras de desigualdad al interior de los countries de Buenos Aires. Ponencia presentada en el XXXI Congreso de la Asociación Latinoamericana de Sociología, Montevideo, Uruguay.

Graham, S. y Marvin, S. (200I). Splintering Urbanism. Networked Infrastructures, Technological Mobilities and the Urban Condition. Routledge.

Gray, E., Farrall, S y Jackson, J. (2007). Experience and Expression; Conversations About Crime, Place and community. Experience and Expression in the Fear of Crime Working Paper, 6, ESRC Grant RES OOO 23 IIO8. 
Harvey, D. (2012). Rebel Cities. From the Rigth to the City to the Urban Revolution. Verso.

Hegel, G.W.F. (2006/1807). Fenomenología del espiritu. Pre-textos.

Hidalgo, M.C. y Hernández B. (200I). Place Attachment: Conceptual and Empirical Questions. Journal of Environmental Psychology, 2I(3), 273-28I.

Hiernaux, D. y Lindón A. (2004a). Repensar la periferia: de la voz a las visiones exo y egocéntricas. Adrián Guillermo Aguilar (coord.), Procesos metropolitanos y grandes ciudades. Dinámicas recientes en México y otros países (4I3-443). unAM, Porrúa.

Hiernaux, D. y Lindón A. (2004b). Desterritorialización y reterritorialización metropolitana: la Ciudad de México, Documents D’anàlisi Geogràfica (44), 7I-88.

Hill, M.K. (2005). Trabajadores y extraños. La economía del servicio doméstico y el panorama del miedo suburbano. En R. Reguillo y M. Godoy Anativia (eds.), Ciudades translocales: espacios, flujo, representación. Perspectivas desde las Américas (20I-235). ITEso, Social Science Research Council (sscr).

Hochschild, A.R. (1979). Emotion Work, Feeling Rules, and Social Structure. American Journal of Sociology, 85(3), 55I-575.

Jackson, J. (20I0). Validating New Measures of the Fear of Crime. International Journal of Social Research Methodology, 8(4), 297-315. $<$ http://eprints.lse.ac.uk/21003/.

Jackson, J., Stephen, F. y Gray, E. (2007). Experience and Expression in the Fear of Crime. Criminal Law eJournal, 44(6). 946-966. doi:IO.2I39/ssrn.IOI2397.

Jacobs, J. (20II/I96I). Muerte y vida de las grandes ciudades. Capitán Swing. Jorquera Limón, R.A. (201I). Ciudad del miedo. La seguridad y el capital social en las clases medias. El Colegio de Sonora.

Kemper, T.D. (1978). Toward a Sociology of Emotions. Some Problems and Some Solutions. The American Sociologist, I3(I), 30-4I.

Kessler, G. (2009). El sentimiento de inseguridad: sociologia del temor al delito. Siglo xxI. 
Kessler, G. y Dimarco, S. (2013). Jóvenes, policía y estigmatización territorial en la periferia de Buenos Aires. Espacio Abierto. Cuaderno Venezolano de Sociología, 22(2), 22I-243.

King, S. (1982). Different Seasons. Viking Press.

Korobov, N. (200I). Reconciling Theory with Method: from Conversation Analysis and Critical Discourse Analysis to Positioning Analysis. Forum Qualitative Sozialforschung/Forum Qualitative Social Research, 2(3). <http://nbn-resolving.de/urn:nbn:de:orI4fqsoio3irg>.

Lara, J. y Mateos, P. (2015). La fragmentación socio-espacial del 'viviendismo': neoliberalismo y desarrollos masivos de vivienda social en la periferia remota de Guadalajara. En A. G. Aguilar, e Escamilla. I. (coords.), Segregación urbana y espacios de exclusión. Ejemplos de México y América Latina (163-193). Instituto de Geografía, Miguel Ángel Porrúa, UNAM.

Lindón, V.A (2005). Figuras de la territorialidad en la periferia metropolitana: topofilias y topofobias. En R. Reguillo y M. Godoy Anativia (eds.), Ciudades translocales: espacios, flujo, representación. Perspectivas desde las Américas (I45-I72). ITESO, Social Science Research Council (sSCR).

Low, S.M. (200I). The Edge and the Center: Gated Communities and the Discourse of Urban Fear", American Anthropologist, I03(I), 45-58. Sampson, R.J. y Raudenbush, S.W. (1999). Systematic Social Observation of Public Spaces: a New Look at Disorder in Urban Neighborhoods. American Journal of Sociology, I05(3), 603-651.

Siqueiros, L.F. (2009a). El territorio, el medio ambiente y las condiciones urbanas. Comisión Nacional para la Prevención y Erradicación de la Violencia contra la Mujer (24-6I). Secretaría de Gobernación.

Siqueiros, L.F. (2009b). El entorno habitacional formal e informal. Comisión Nacional para la Prevención y Erradicación de la Violencia contra la Mujer (62-96). Secretaría de Gobernación.

Stets, J.E. (2012). Current Emotion Research in Sociology: Advances in the Discipline. Emotion Review, 4(3), 326-334.

Skogan, W. (1986). Fear of Crime and Neighborhood Change. Crime and Justice, 8, 203-229. 
Svampa, M. (2004). Fragmentación espacial y procesos de integración social 'hacia arriba'. Socialización, sociabilidad y ciudadanía. Espiral, Estudios de Estado y Sociedad, XI(3I), 55-84.

Tabachnick, B.G. y. Fidell, L.S. (1996). Using Multivariate Statistics (3a. ed.). Harper Collins.

Tulloch, M. (2003). Combining Classificatory and Discursive Methods: Consistency and Variability in Responses to Threat of Crime. British Journal of Social Psychology, 42 (3), 46I-476. <https://doi. org/IO.I348/oI4466603322438260>.

Unikel, L. (1976). El desarrollo urbano en México, diagnóstico e implicaciones futuras. El Colegio de México.

Veblen, T. (2009/1899). The Theory of the Leisure Class. Oxford: Oxford University Press.

Wacquant, L. (200Ia). The Rise of Advanced Marginality: Notes on its Nature and Implications, Acta Sociológica (39), I2I-I39. < https:// doi.org/Io.II77/ooor6993960390020I >.

Wacquant, L. (200Ib). Parias urbanos. Marginalidad en la ciudad a comienzos del milenio, Manantial.

Wacquant, L. (2007). Territorial Stigmatization in the Age of Advanced Marginality. Thesis Eleven, $9 I(\mathrm{I}), 66-77$.

Wacquant, L. (20II). Desolación urbana y denigración simbólica en el hiperguetto. Astrolabio, Nueva Época (6), 4-I8.

Wooffitt, R. (2005). Conversation Analysis and Discourse Analysis. A Comparative and Critical Introduction. Sage Publications.

Young, M. y Willmott P. (1962). Keeping Themselves to Themselves. En E. Josephson, M. Josephson (comps.), Man Alone: Alienation in Modern Society(312-326). New York: Dell. 


\section{ANEXO}

Tabla AI. Medias y desviaciones estándar de otros indicadores relevantes

\begin{tabular}{|c|c|c|c|}
\hline Clúster & $\begin{array}{l}\text { edad de persona } \\
\text { entrevistada }\end{array}$ & Tamańo del hogar & Ingreso per cápita mensual \\
\hline \multirow[t]{2}{*}{ I } & 37.6 & 5 & 4640.114 \\
\hline & I2.6 & 2.04 & 3432.182 \\
\hline \multirow[t]{2}{*}{2} & 40.3 & 3.48 & $6036.57 \mathrm{I}$ \\
\hline & 16.2 & I.I8 & 5188.857 \\
\hline \multirow[t]{2}{*}{3} & 38.4 & 4.18 & 49I4.I5I \\
\hline & I2.8 & I.I6 & 2094.102 \\
\hline \multirow[t]{2}{*}{ Total } & 39.3 & 3.97 & 5471.109 \\
\hline & I4.56 & I. 52 & 4284.962 \\
\hline
\end{tabular}

Percepción del progreso en el fraccionamiento:

Para producir los datos que se incluyen en la siguiente tabla, se incluyó esta pregunta en el cuestionario de la encuesta a hogares:

En comparación con el año pasado, ¿̨usted diría que en estos aspectos su fraccionamiento está...?2

_ Mejor que antes

_ Igual que antes

_ Peor que antes

_ [Sí «mejor que antes» o "peor que antes»]. Por favor, descríbame en qué ha cambiado su colonia o fraccionamiento en lo que se refiere a estos aspectos que le acabo de preguntar

\footnotetext{
${ }^{2}$ Pregunta formulada así con base en Jackson et al. (2007).
} 
Tabla A2. Percepción de progreso (o deterioro) del fraccionamiento en relación con eficacia colectiva, respeto, apoyo mutuo

\begin{tabular}{lccccc}
\hline & \multicolumn{3}{c}{ Cluster } & & \\
\cline { 2 - 5 } & I & $\mathbf{2}$ & $\mathbf{3}$ & Total \\
\hline Mejor que antes & 6 & 4 & 6 & I6 \\
\hline Igual que antes & 5 & I6 & 4 & 25 \\
\hline Peor que antes & 0 & 7 & I & 8 \\
\hline Total & II & 27 & II & 49 \\
\hline Pearson chi2 $(4)=$ I0.4220 $\operatorname{Pr}=0.034$ & & & & \\
\hline
\end{tabular}

Deseos de mudanza (sueños de fuga):

En nuestra investigación se usó la frase «si pudiera, me mudaba de este lugar». ${ }^{3}$

Tabla A3. Deseos de mudarse (¿Qué tan de acuerdo está con la frase «Si pudiera, me mudaba de aquím?)

\begin{tabular}{|c|c|c|c|c|}
\hline & \multicolumn{4}{|c|}{ Cluster } \\
\hline & $\mathbf{I}$ & 2 & 3 & Total \\
\hline Totalmente de acuerdo & o & I3 & 4 & I7 \\
\hline De acuerdo & o & 7 & 4 & II \\
\hline Ni de acuerdo ni en desacuerdo & o & 3 & I & 4 \\
\hline En desacuerdo & o & 4 & 2 & 6 \\
\hline Totalmente en desacuerdo & II & o & o & II \\
\hline Total & II & 27 & II & 49 \\
\hline
\end{tabular}

Pearson $\operatorname{chi} 2(8)=49.8036 \quad \operatorname{Pr}=0.000$

${ }^{3}$ Tomando como referencia los estudios de Conklin (197I); Sampson y Raudenbush (1999); Fidel, Di Tomasso y Farías (2008); Gerson et al., 1977, cit. en Hidalgo y Hernández (200I, p. 276). 